\title{
An Outage-Based Game Design for Cognitive Radio
}

\author{
Ehsan Nekouei, Tansu Alpcan and Subhrakanti Dey \\ Department of Electrical and Electronic Engineering \\ The University of Melbourne, VIC., 3010, Australia
}

\begin{abstract}
This paper addresses the power control problem in an underlay cognitive radio network where $N$ selfish secondary users (SUs) sharing a frequency band with a primary user. The problem is studied from two perspectives: system level and user level. At the system level, a secondary network manager (SNM) is interested in minimizing the secondary network outage probability subject to peak power constraints on SU transmission power levels and a constraint on the maximum interference generated at the primary receiver. The resulting system level optimization problem is converted to a convex one. At the user level, SUs selfishly increase their transmission power levels to maximize their outage-based utility functions, and SNM imposes a cost on each $\mathrm{SU}$ based on its interference power at the primary receiver. The system level optimization problem is converted to a convex one. The user level optimization problem is formulated as a strategic (non-cooperative) power control game, which admits a unique Nash equilibrium. A distributed algorithm for computing the Nash equilibrium is proposed. Finally, an optimal pricing scheme is presented which eliminates the gap between network and user level performances by aligning the optimal solution of system level problem with the Nash equilibrium of the user level problem.
\end{abstract}

\section{INTRODUCTION}

Cognitive radio technologies aim to alleviate frequency spectrum scarcity by enabling unlicensed users, alternatively called secondary users or SUs, to transmit at the same frequency spectrum with legacy users, alternatively called primary users or PUs, as long as the SUs do not cause harmful interference at primary receivers. Different mechanism for coexistence of SUs and PUs has been studied such as underlay, overlay and interweave [1]. In the underlay paradigm, the paradigm of interest in this paper, SUs transmit concurrently with PUs, but they should keep their interference temperature at the primary network below a predetermined level [1].

Game theory provides a flexible framework for modeling the interaction of selfish agents competing for shared resources. Game theoretic solutions has been extensively studied for power control problem in wireless networks in the past decade. Among many results, authors in [2] studied existence, uniqueness and efficiency of Nash equilibria in energy efficient power control games for wireless networks. In [3], authors considered an outage game for multi-cell uplink CDMA networks. They studied uniqueness of Nash equilibrium as well as different equilibrium seeking power update algorithms. It is well known that Nash equilibria are inefficient in general, that is, the game outcome at Nash equilibria is not generally compatible with performance of the centralized solution [4] which implies that power control games in wireless networks are in general in inefficient. In [5], Alpcan et al. proposed a pricing approach for closing the gap between the game outcome and the centralized solution under fairly general conditions on the game utility and the price functions.

Game theory has been considered for power control problem in cognitive radio network. In [6], authors studied a game theoretic approach for minimizing SUs' transmission powers subject to outage probability constraints at secondary and primary receivers. In [7], authors considered a repeated power control game with cheat proof strategies for SUs competing for transmission in unlicensed bands.

In this paper, we consider a secondary network with $N$ secondary transmitter-receiver pairs communicating at the same frequency with a primary user. We study the power control problem in a conflicting situation in which secondary network manager (SNM) concerns about interference management task and social optimality of power control problem, but secondary users (SUs) are selfish decision-makers concerning about maximizing their utility functions. We model this situation as a two level optimization problem: system level and user level. In the system level, SNM concerns about minimizing secondary network outage probability when transmission power of SUs are limited by peak power constraints as well as a constraint on the interference power of secondary network at the primary receiver. In the user level, SUs selfishly decide about their transmission power levels in order to maximize their outagebased utility functions, and SNM imposes a cost on the utility of each SU based on its interference power at the primary receiver.

- We show that system level optimization problem can be converted to a convex optimization problem.

- The user level optimization problem is modeled as a strategic non-cooperative power control game. We show that this game admits a unique Nash equilibrium, and a distributed algorithm for computing Nash equilibrium is proposed.

- We also derive the optimal interference pricing scheme which aligns the system level solution as the Nash equilibrium of user level problem.

The rest of the paper is organized as follows. Section II describes the network model along with system level optimization problem. Section III describes user level optimization problems along with game design. Section IV presents some numerical results to illustrate the performance of the pricing scheme in aligning the system level and user level solutions. Section $\mathrm{V}$ concludes the paper. 


\section{System LeVel Optimization Problem}

Consider a cognitive radio network with $N$ secondary transmitter-receiver pairs sharing a frequency band with a PU. Secondary transmitter (ST) $i$ communicates with secondary receiver (SR) $i$, and at the same time, interferes with signal reception at other SRs as well as the primary receiver. Let $H_{i j}^{s s}$ and $G_{i j}^{s s}$ represent fast fading and slow fading components of channel power gain between ST- $i$ and SR- $j$, respectively. Let $H_{i, 1}^{s p}$ and $G_{i 1}^{s p}$ represent fast fading and slow fading components of channel power gain between ST $i$ and the primary receiver. Slow fading components of secondary-transmitter-secondary-receiver (STSR) and secondary-transmitter-primary-receiver (STPR) channel power gains, i.e., $\left\{G_{i j}^{s s}\right\}_{i, j=1}^{N}$ and $\left\{G_{i 1}^{s p}\right\}_{i}^{N}$, are assumed to be fixed during the time-scale of interest in this paper. We assume that fast fading components of STSR and STPR channel power gains, i.e., $\left\{H_{i j}^{s s}\right\}_{i, j=1}^{N}$ and $\left\{H_{i 1}^{s p}\right\}_{i}^{N}$, be independent and exponentially distributed random variables with unit mean.

We consider a scenario in which SUs are not able to track fast fading components of channel gains, hence, they allocate their transmission powers according to slow fading components. In this scenario, a proper measure of secondary network QoS is the outage probability. Let $\mathrm{SINR}_{i}$ be the signal to interference plus noise ratio (SINR) of SR- $i$ which is given by

$$
\operatorname{SINR}_{i}=\frac{G_{i i}^{s s} H_{i i}^{s s} P_{i}}{\sigma^{2}+\sum_{j=1, j \neq i}^{N} G_{j i}^{s s} H_{j i}^{s s} P_{j}},
$$

where $P_{j}$ is the transmission power of ST- $j$ and $\sigma^{2}$ is the additive noise power at SR-i. We define the network outage probability, $O\left(P_{1}, \cdots, P_{N}\right)$, as

$$
\begin{aligned}
O\left(P_{1}, \cdots, P_{N}\right) & =\operatorname{Pr}\left(\operatorname{SINR}_{N}^{\star} \leq \gamma_{\mathrm{th}}\right) \\
& =1-\prod_{i=1}^{N}\left(1-\operatorname{Pr}\left(\operatorname{SINR}_{i} \leq \gamma_{\mathrm{th}}\right)\right) \\
& =1-\prod_{i=1}^{N}\left(1-O_{i}\left(P_{1}, \cdots, P_{N}\right)\right),
\end{aligned}
$$

where $\operatorname{SINR}_{N}^{\star}=\min _{i} \operatorname{SINR}_{i}, \gamma_{\text {th }}$ is a a predetermined threshold value and $O_{i}\left(P_{1}, \cdots, P_{N}\right)=\operatorname{Pr}\left(\operatorname{SINR}_{i} \leq \gamma_{\text {th }}\right)$ is the outage probability of SU- $i$. Network outage probability represents the fraction of time that at least SINR of one SU falls below $\gamma_{\text {th }}$. Following the results of [8], it is easy to show that for Rayleigh distributed STSR channel gains, outage probability of $\mathrm{SU}-i$ is given by:

$$
O_{i}\left(P_{1}, \cdots, P_{N}\right)=1-\mathrm{e}^{-\frac{\sigma^{2} \gamma_{\mathrm{th}}}{G_{i i}^{s i t}}} \prod_{i=1, j \neq i}^{N} \frac{1}{1+\frac{\gamma_{\mathrm{th}} G_{j i}^{s s} P_{j}}{G_{i i}^{s s} P_{i}}} .
$$

Hence, $O\left(P_{1}, \cdots, P_{N}\right)$ can be written as

$O\left(P_{1}, \cdots, P_{N}\right)=1-\prod_{i=1}^{N}\left(\mathrm{e}^{-\frac{\sigma^{2} \gamma_{\mathrm{th}}}{G_{i i}^{S i} P_{i}}} \prod_{j=1, j \neq i}^{N} \frac{1}{1+\frac{\gamma_{\mathrm{th}} G_{j i}^{s s} P_{j}}{G_{i i}^{s s} P_{i}}}\right)$
In the system level, the SNM is interested in minimizing the secondary network outage probability when transmission powers of SUs are limited by peak power constraints and a constraint on the average interference that they cause to the primary receiver. That is, in the system level, transmission powers of SUs are given by the solution of following optimization problem:

$$
\begin{array}{ll}
\underset{\begin{array}{c}
P_{1}, \cdots, P_{N} \\
\text { subject to : }
\end{array}}{\operatorname{minimize}} & O\left(P_{1}, \cdots, P_{N}\right) \\
& 0 \leq P_{i} \leq P_{\mathrm{m}}, \quad i=1, \cdots, N \\
& \sum_{i=1}^{N} G_{i 1}^{s p} P_{i} \leq Q_{\mathrm{m}},
\end{array}
$$

where $P_{\mathrm{m}}$ and $Q_{\mathrm{m}}$ are the peak power and peak interference constraints, respectively. Note that the interference constraint represents the average interference at the primary receiver where the average is taken over fast fading components of STPR channel power gains. The constraints in (4) are convex, but the objective function is complicated. To simplify the objective function, we define the following equivalent problem:

$$
\begin{array}{ll}
\underset{\begin{array}{c}
P_{1}, \cdots, P_{N} \\
\text { subject to : }
\end{array}}{\operatorname{minimize}} & -\log \left(1-O\left(P_{1}, \cdots, P_{N}\right)\right) \\
& 0 \leq P_{i} \leq P_{\mathrm{m}}, \quad i=1, \cdots, N \\
& \sum_{i=1}^{N} G_{i 1}^{s p} P_{i} \leq Q_{\mathrm{m}} .
\end{array}
$$

The objective function in (5) can be expressed as

$$
\sum_{i=1}^{N} \frac{\sigma^{2} \gamma_{\mathrm{th}}}{G_{i i}^{s s} P_{i}}+\log \left(\prod_{i=1}^{N} \prod_{j=1, j \neq i}^{N} 1+\frac{\gamma_{\mathrm{th}} G_{j i}^{s s} P_{j}}{G_{i i}^{s s} P_{i}}\right) .
$$

Note that $\prod_{i=1}^{N} \prod_{j=1, j \neq i}^{N} 1+\frac{\gamma_{\mathrm{th}} G_{j i}^{s s} P_{j}}{G_{j i}^{s s} P_{i}}$ is a posynomial [9], hence, the objective function in (5) can be convexified using the transformation $P_{i}=\mathrm{e}^{\hat{P}_{i}}$. We define the new optimization problem as:

$$
\begin{array}{cl}
\underset{\hat{P}_{1}, \cdots, \hat{P}_{N}}{\operatorname{minimize}} & -\log \left(1-O\left(\mathrm{e}^{\hat{P}_{1}}, \cdots, \mathrm{e}^{\hat{P_{N}}}\right)\right) \\
\text { subject to }: & \mathrm{e}^{\hat{P}_{i}} \leq P_{\mathrm{m}}, \quad i=1, \cdots, N \\
& \sum_{i=1}^{N} G_{i 1}^{s p} \mathrm{e}^{\hat{P}_{i}} \leq Q_{\mathrm{m}} .
\end{array}
$$

The objective function in (7) is convex, and the constraints are convex. Hence, (7) can be solved using efficient numerical algorithms.

\section{Game Design And User Level Optimization PROBLEM}

In the user level, we consider a scenario in which SUs selfishly choose their transmission power levels to minimize their outage probabilities, but SNM imposes a cost on each SUs based on its average interference power at the primary receiver. This pricing scheme assists SNM to guarantee a level of QoS in the secondary network, and also control the amount of secondary network interference at the primary receiver. We model this scenario as a strategic non-cooperative power control (SNPC) game in which SUs simultaneously and independently determine their transmission powers levels. The strategy of SU-i is its transmission power level $P_{i}$, and 
its action set is $\mathcal{P}_{i}=\left[0, P_{m}\right]$. The utility function of SU- $i$, $U_{i}\left(P_{i}, \boldsymbol{P}_{-i}\right)$, is defined as

$$
U_{i}\left(P_{i}, \boldsymbol{P}_{-i}\right)=\log \left(1-O_{i}\left(P_{i}, \boldsymbol{P}_{-i}\right)\right)-\mu_{i} G_{i 1}^{s p} P_{i},
$$

where $\mu_{i}$ is a positive number representing the price per unit interference power that SU- $i$ causes at the primary receiver, and $\boldsymbol{P}_{-i}$ is strategies of all SUs expect SU- $i$. Note that the term $\log \left(1-O_{i}\left(P_{i}, \boldsymbol{P}_{-i}\right)\right)$ in (8) is a metric for the QoS of SU-i. Let $\mathcal{P}^{N}=\mathcal{P}_{1} \times \cdots \times \mathcal{P}_{N}$ and $\mathcal{N}=\{1, \cdots, N\}$. We refer to this game as $\left\langle\mathcal{N},\left\{\mathcal{P}_{i}\right\},\left\{U_{i}(\cdot)\right\}\right\rangle$. The user level optimization problem can be expressed as

$$
\max _{P_{i} \in \mathcal{P}_{i}} U_{i}\left(P_{i}, \boldsymbol{P}_{-i}\right) \quad \forall i \in \mathcal{N} .
$$

Different solution concepts has been considered for strategic games which among them, Nash equilibrium is the most widely accepted solution concept. $\boldsymbol{P}^{\mathrm{NE}} \in \mathcal{P}^{N}$ is a Nash equilibrium of the SNPC game $\left\langle\mathcal{N},\left\{\mathcal{P}_{i}\right\},\left\{U_{i}(\cdot)\right\}\right\rangle$ if, for all $i \in \mathcal{N}$, we have

$$
U_{i}\left(P_{i}^{\mathrm{NE}}, \boldsymbol{P}_{-i}^{\mathrm{NE}}\right) \geq U_{i}\left(P_{i}, \boldsymbol{P}_{-i}^{\mathrm{NE}}\right) \quad \forall P_{i} \in \mathcal{P}_{i} .
$$

Hence, knowing that other SUs play their Nash equilibrium strategies, no SU can increase its utility by unilaterally deviating its transmission power level from the equilibrium strategy. But, in general, a game may not have a Nash equilibrium. In the next Theorem, we study existence and uniqueness of Nash equilibrium of the SNPC game.

Theorem 1: The SNPC game $\left\langle\mathcal{N},\left\{\mathcal{P}_{i}\right\},\left\{U_{i}(\cdot)\right\}\right\rangle$ admits a unique Nash equilibrium.

Proof: Please see Appendix A.

In general, computing Nash equilibria of a game is a complex problem, and efficient algorithms of computing Nash equilibria for different game types is an active research area, e.g., see [10] and references therein. Here, we introduce a distributed algorithm for computing unique Nash equilibrium of the SNPC game $\left\langle\mathcal{N},\left\{\mathcal{P}_{i}\right\},\left\{U_{i}(\cdot)\right\}\right\rangle$. We refer to this algorithm as Algorithm 1. Initially, Algorithm 1 sets transmission powers of all SUs to zero. At $n$th iteration of the algorithm, for all $i \in \mathcal{N}$, SU- $i$ declares its strategy $P_{i}^{(n)}$ to other SUs, and constructs strategy vector of other SUs, $\boldsymbol{P}_{-i}^{(n)}$. Then, SU- $i$ will compute its best response to $\boldsymbol{P}_{-i}^{(n)}, B_{i}\left(\boldsymbol{P}_{-i}^{(n)}\right)$, which is given by $B_{i}\left(\boldsymbol{P}_{-i}^{(n)}\right)=\min \left(P_{m}, F_{i}\left(\boldsymbol{P}_{-i}^{(n)}\right)\right)$ where $F_{i}\left(\boldsymbol{P}_{-i}^{(n)}\right)$ is obtained by solving the following equation for $P_{i}$ (see Appendix A for more details)

$$
\frac{\sigma^{2} \gamma_{\mathrm{th}}}{G_{i i}^{s s} P_{i}^{2}}+\frac{1}{P_{i}} \sum_{j=1, j \neq i}^{N} \frac{\gamma_{\mathrm{th}} G_{j i}^{s s} P_{j}^{(n)}}{G_{i i}^{s s} P_{i}+\gamma_{\mathrm{th}} G_{j i}^{s s} P_{j}^{(n)}}=\mu_{i} G_{i i}^{s p} .
$$

Then, SU- $i$ sets $B_{i}\left(\boldsymbol{P}_{-i}^{(n)}\right)$ as its strategy for the next iteration of the Algorithm.

We note that Algorithm 1 just requires massage passing among the SUs. In this next lemma, we establish that under Algorithm 1, transmission powers of SUs converge to the Nash equilibrium.

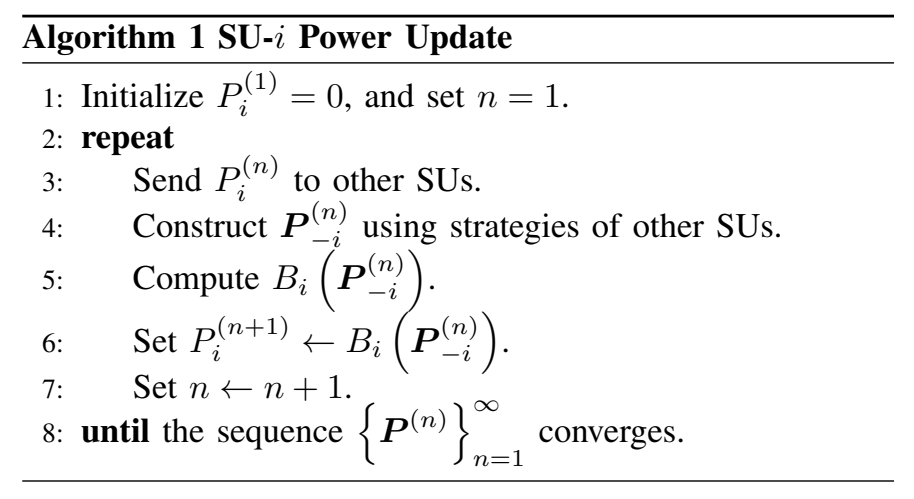

Theorem 2: Let $\boldsymbol{P}^{(n)}$ be the strategy vector of SUs at $n$ th iteration of Algorithm 1. Then, the sequence $\left\{\boldsymbol{P}^{(n)}\right\}_{n=1}^{\infty}$ converges to the unique Nash equilibrium of the SNPC game $\left\langle\mathcal{N},\left\{\mathcal{P}_{i}\right\},\left\{U_{i}(\cdot)\right\}\right\rangle$.

Proof: Please see Appendix B.

At each iteration of Algorithm 1, SU-i solves the nonlinear equation (11) for $P_{i}$ in order to obtain its best response to $\boldsymbol{P}_{-i}^{(n)}$. In the next Lemma, we propose a fixed point iteration for solving (11).

Lemma 1: Let $\left\{\pi_{m}(i)\right\}_{m=1}^{\infty}$ be the sequence generated by fixed point iteration $\pi_{m+1}=T_{i}\left(\pi_{m}(i), \boldsymbol{P}_{-i}^{(n)}\right)$ with $\pi_{1}(i)=0$ where $T_{i}\left(\pi_{m}(i), \boldsymbol{P}_{-i}^{(n)}\right)$ is given by (12). Then, $\left\{\pi_{m}(i)\right\}_{m=1}^{\infty}$ converge to the solution of (11).

Proof: Please see Appendix C.

Remark 1: In the process of computing fixed point iteration $\pi_{m+1}=T_{i}\left(\pi_{m}(i), \boldsymbol{P}_{-i}^{(n)}\right)$, we follow the usual convention that $\frac{1}{0}=\infty, \frac{1}{\infty}=0$.

\section{A. Game Design}

In this subsection, we derive the optimal interference pricing scheme which aligns the solution of network and user level optimization problems. Let $\boldsymbol{P}^{\star}$ be the solution of optimization problem (4), and $\boldsymbol{\mu}^{\star}=\left[\mu_{1}^{\star}, \cdots, \mu_{N}^{\star}\right]^{\top}$ be the optimal interference price vector. For $\boldsymbol{P}^{\star}$ to be the Nash equilibrium of the SNPC game $\left\langle\mathcal{N},\left\{\mathcal{P}_{i}\right\},\left\{U_{i}(\cdot)\right\}\right\rangle$ with pricing scheme $\boldsymbol{\mu}^{\star}, P_{i}^{\star}$ should be the best response of SU-i to $\boldsymbol{P}_{-i}^{\star}$, i.e.,

$$
\frac{\sigma^{2} \gamma_{\mathrm{th}}}{G_{i i}^{s s} P_{i}^{\star 2}}+\frac{1}{P_{i}^{\star}} \sum_{j=1, j \neq i}^{N} \frac{\gamma_{\mathrm{th}} G_{j i}^{s s} P_{j}^{\star}}{G_{i i}^{s s} P_{i}^{\star}+\gamma_{\mathrm{th}} G_{j i}^{s s} P_{j}^{\star}}=\mu_{i}^{\star} G_{i i}^{s p},
$$

for all $i \in \mathcal{N}$. Hence, the optimal interference price of SU-i, $\mu_{i}^{\star}$, is given by

$\mu_{i}^{\star}=\frac{\gamma_{\mathrm{th}}}{G_{i i}^{s p}}\left(\frac{\sigma^{2}}{G_{i i}^{s s} P_{i}^{\star 2}}+\frac{1}{P_{i}^{\star}} \sum_{j=1, j \neq i}^{N} \frac{G_{j i}^{s s} P_{j}^{\star}}{G_{i i}^{s s} P_{i}^{\star}+\gamma_{\mathrm{th}} G_{j i}^{s s} P_{j}^{\star}}\right)$.

According to (14), optimal interference price of SU- $i$ is inversely proportional to slow component of its STPR channel power gain which implies that SNM imposes high interference price on SUs with small STPR channel power gains to avoid severe interference at other SRs. Note that, with a flat pricing scheme, SUs with small STPR channel power gains transmit 


$$
T_{i}\left(\pi_{m}(i), \boldsymbol{P}_{-i}^{(n)}\right)=\sqrt{\frac{\gamma_{\mathrm{th}}}{\mu_{i} G_{i i}^{s p}}}\left(\frac{\sigma^{2}}{G_{i i}^{s s}}+\sum_{j=1, j \neq i}^{N} \frac{G_{j i}^{s s}}{\frac{G_{i i}^{s s}}{P_{j}^{(n)}}+\frac{\gamma_{\mathrm{th}} G_{j i}^{s s}}{\pi_{m}(i)}}\right)^{0.5} .
$$

with high powers which results in higher outage probability compare to optimal pricing scheme. Employing optimal pricing scheme also guarantees that the interference constraint at the primary receiver is respected at the Nash equilibrium.

\section{NUMERICAL STUDY}

In this section, we present our numerical results for a cognitive radio network with 4 secondary transmitter-receiver pairs. We model the slow fading component of fading power gain between a secondary transmitter and a secondary/primary receiver as $\left(\frac{0.1}{d}\right)^{2.5} Y^{-1}$ where $d$ is distance between the corresponding transmitter and receiver, and $\log (Y)$ is a Gaussian distributed random variable with zero mean and standard deviation 0.1 . We also assume that the $d$ is uniformly distributed over $[0,1000]$. The noise power $\sigma^{2}$ is assumed to be $10^{-13}$. First, SNM computes the optimal power levels, and the optimal interference price of each SU. Then, SUs will be notified about their interference prices. Then, SUs employ Algorithm 1 to compute their Nash equilibrium strategies.

Fig. 1 shows the power update of SUs for 10 iterations of Algorithm 1. In Fig. 1, we set $P_{\mathrm{m}}, Q_{\mathrm{m}}$ and $\gamma_{\text {th }}$ to $10 \mathrm{~dB}$, $0 \mathrm{~dB}$ and $10 \mathrm{~dB}$, respectively. Similar quantitative behavior continue to hold for other values of $P_{\mathrm{m}}, Q_{\mathrm{m}}$ and $\gamma_{\mathrm{th}}$. As Fig. 1 shows, using Algorithm 1 with $\boldsymbol{P}^{(1)}=\mathbf{0}$, transmission power of SUs converge to their Nash equilibrium strategies as predicted by Theorem 2. It also shows that using the optimal pricing scheme, SNM is able to align the optimal power levels to the Nash equilibrium of the power control game, and hence, control the behavior of selfish SUs. Finally, as Fig. 1 shows, the output of Algorithm 1 with $\boldsymbol{P}^{(1)}=\mathbf{0}$ constitute an increasing sequence of power updates as predicted by Lemma 2 in Appendix B.

\section{CONCLUSION}

In this paper, we studied the problem of power control in cognitive radio networks from system level and user level point of views. In the system level, we considered a scenario in which secondary network manager is interested in minimizing the network outage probability when transmission powers of SUs are limited by peak power constraints and a constraint on the interference power of secondary network at the primary receiver. In the user level, we considered a scenario in which SUs selfishly decide their transmission power level to maximize their utility functions. We showed that the system level problem can be transfered to a convex optimization problem. We also modeled the user level problem as a strategic noncooperative power control game which admits a unique a unique Nash equilibrium. A distributed algorithm for computing the Nash equilibrium is also proposed. Finally, we derived
Power Updates of STs according to Algorithm 1

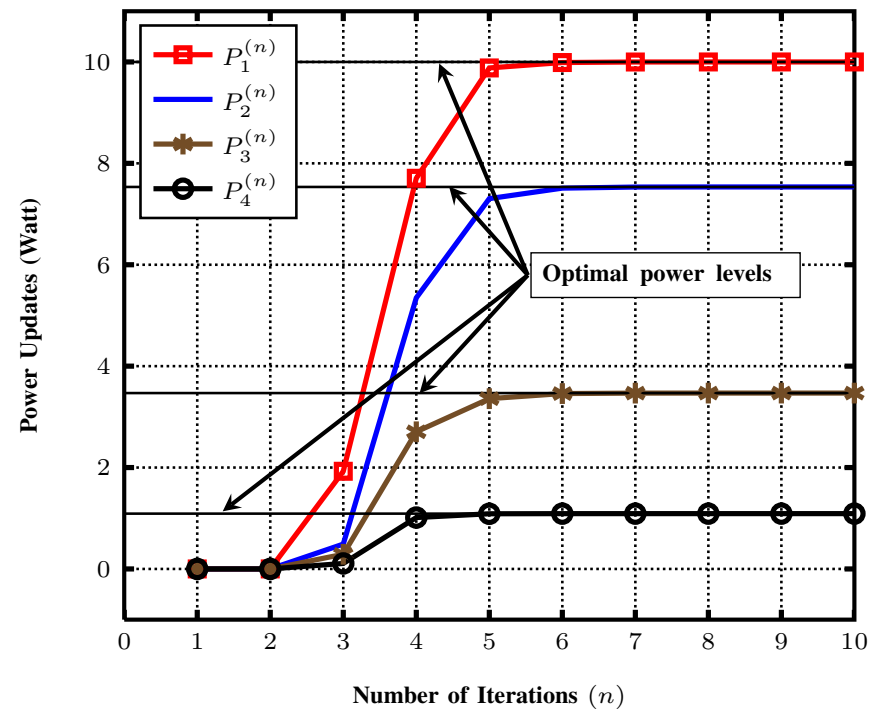

Fig. 1. Secondary network throughput scaling under

the optimal pricing scheme which enables secondary network manager to close the gap between the network performance at user level and system level solutions.

\section{APPENDIX A}

We start the proof by showing that the SNPC game $\left\langle\mathcal{N},\left\{\mathcal{P}_{i}\right\},\left\{U_{i}(\cdot)\right\}\right\rangle$ admits a Nash equilibrium. Then, we show that the Nash equilibrium is unique. Note that the action set of SU-i, $\mathcal{P}_{i}=\left[0, P_{m}\right]$, is a non-empty, closed and bounded subset of $\mathbb{R}$. Hence, it is compact by Heine-Borel Theorem [11]. $\mathcal{P}_{i}$ is also convex. $U_{i}\left(P_{i}, \boldsymbol{P}_{-i}\right)$ is a continuous function in $P_{i}$. It is easy to show that $U_{i}\left(P_{i}, \boldsymbol{P}_{-i}\right)$ is concave with respect to $P_{i}$. Hence, the SNPC game $\left\langle\mathcal{N},\left\{\mathcal{P}_{i}\right\},\left\{U_{i}(\cdot)\right\}\right\rangle$ admits a Nash equilibrium [12].

To show the uniqueness of Nash equilibrium, we exploit the structure of best response function of each SU given strategies of other SUs. Given $\boldsymbol{P}_{-i}$, let $F_{i}\left(\boldsymbol{P}_{-i}\right): \mathcal{P}^{N-1} \rightarrow \mathbb{R}_{+}$be the solution of unconstrained version of optimization problem in (9), i.e., $F_{i}\left(\boldsymbol{P}_{-i}\right)$ satisfies the first order optimality condition:

$$
\begin{array}{r}
\frac{\sigma^{2} \gamma_{\mathrm{th}}}{G_{i i}^{s s} F_{i}\left(\boldsymbol{P}_{-i}\right)^{2}}+\frac{1}{F_{i}\left(\boldsymbol{P}_{-i}\right)} \sum_{j=1, j \neq i}^{N} \frac{\gamma_{\mathrm{th}} G_{j i}^{s s} P_{j}}{G_{i i}^{s s} F_{i}\left(\boldsymbol{P}_{-i}\right)+\gamma_{\mathrm{th}} G_{j i}^{s s} P_{j}} \\
=\mu_{i} G_{i i}^{s p} .
\end{array}
$$

Note that $F_{i}\left(\boldsymbol{P}_{-i}\right)$ is the best response of SU- $i$ to $\boldsymbol{P}_{-i}$ if $F_{i}\left(\boldsymbol{P}_{-i}\right)$ is feasible, i.e. $F_{i}\left(\boldsymbol{P}_{-i}\right) \in \mathcal{P}_{i}$. It 
is easy to show that $\frac{\partial U_{i}\left(P_{i}, \boldsymbol{P}_{-i}\right)}{\partial P_{i}} \geq 0$ for $P_{i} \leq$ $F_{i}\left(\boldsymbol{P}_{-i}\right)$. Hence, the best response of SU-i, $B_{i}\left(\boldsymbol{P}_{-i}\right)$, to $\boldsymbol{P}_{-i}$ is given by $B_{i}\left(\boldsymbol{P}_{-i}\right)=\min \left(P_{m}, F_{i}\left(\boldsymbol{P}_{-i}\right)\right)$. Let $\boldsymbol{F}(\boldsymbol{P})=\left[F_{1}\left(\boldsymbol{P}_{-1}\right), \cdots, F_{N}\left(\boldsymbol{P}_{-N}\right)\right]^{\top}$ and $\boldsymbol{B}(\boldsymbol{P})=$ $\left[B_{1}\left(\boldsymbol{P}_{-1}\right), \cdots, B_{N}\left(\boldsymbol{P}_{-N}\right)\right]^{\top}$.

A strategy vector $\boldsymbol{P} \in \mathcal{P}^{N}$ is a unique Nash equilibrium of the SNPC game if and only if $\boldsymbol{P}$ is a unique fixed point of $\boldsymbol{B}(\boldsymbol{P})$. Thus, the proof is complete if we show that $\boldsymbol{B}(\boldsymbol{P})$ has a unique fixed point. To this end, we show that $\boldsymbol{F}(\boldsymbol{P})$ and $\boldsymbol{B}(\boldsymbol{P})$ are standard functions [13]. $\boldsymbol{F}(\boldsymbol{P})$ is a standard function if it satisfies the following properties for all $\boldsymbol{P} \succ \mathbf{0}$ :

- Positivity: $\boldsymbol{F}(\boldsymbol{P}) \succ \mathbf{0}$.

- Monotonicity: $\boldsymbol{F}\left(\boldsymbol{P}_{1}\right) \succeq \boldsymbol{F}\left(\boldsymbol{P}_{2}\right)$ for $\boldsymbol{P}_{\mathbf{1}} \succeq \boldsymbol{P}_{\mathbf{2}}$.

- Scalability: $\boldsymbol{F}(\alpha \boldsymbol{P}) \prec \alpha \boldsymbol{F}(\boldsymbol{P})$ for $\alpha>1$.

To show the positivity of $\boldsymbol{F}(\boldsymbol{P})$, we note that the left hand side of (15) is decreasing with $F_{i}\left(\boldsymbol{P}_{-i}\right)$ for $\boldsymbol{P}_{-i} \succ \mathbf{0}$. It also tends to zero and infinity as $F_{i}\left(\boldsymbol{P}_{-i}\right)$ tends to infinity and zero, respectively. Hence, $F_{i}\left(\boldsymbol{P}_{-i}\right)$ is the positive and unique solution of (15) which implies $\boldsymbol{F}(\boldsymbol{P}) \succ \mathbf{0}$. Monotonicity of $\boldsymbol{F}(\boldsymbol{P})$ directly follows from (15). To show that $\boldsymbol{F}(\boldsymbol{P})$ is scalable, we note that $F_{i}\left(\alpha \boldsymbol{P}_{-i}\right)$ is the solution of following equation for $P_{i}$

$$
\frac{\sigma^{2} \gamma_{\mathrm{th}}}{G_{i i}^{s s} P_{i}^{2}}+\frac{1}{P_{i}} \sum_{j=1, j \neq i}^{N} \frac{\gamma_{\mathrm{th}} G_{j i}^{s s} \alpha P_{j}}{G_{i i}^{s s} P_{i}+\gamma_{\mathrm{th}} G_{j i}^{s s} \alpha P_{j}}=\mu_{i} G_{i i}^{s p} .
$$

For $\alpha>1, \alpha F_{i}\left(\boldsymbol{P}_{-i}\right)$ can not be the solution of (16) since $\frac{\sigma^{2} \gamma_{\mathrm{th}}}{G_{i i}^{s s} \alpha^{2} F_{i}\left(\boldsymbol{P}_{-i}\right)^{2}}+$

$$
\frac{1}{\alpha F_{i}\left(\boldsymbol{P}_{-i}\right)} \sum_{j=1, j \neq i}^{N} \frac{\gamma_{\mathrm{th}} G_{j i}^{s s} \alpha P_{j}}{G_{i i}^{s s} \alpha F_{i}\left(\boldsymbol{P}_{-i}\right)+\gamma_{\mathrm{th}} G_{j i}^{s s} \alpha P_{j}}<\mu_{i} G_{i i}^{s p} .
$$

This observation implies that any real number greater or equal to $\alpha F_{i}\left(\boldsymbol{P}_{-i}\right)$ can not be the solution of (16) for $\alpha>1$. Hence, $F_{i}(\alpha \boldsymbol{P})<\alpha F_{i}\left(\boldsymbol{P}_{-i}\right)$ and $\boldsymbol{F}(\alpha \boldsymbol{P}) \prec \alpha \boldsymbol{F}(\boldsymbol{P})$ for $\alpha>1$. This implies that $\boldsymbol{F}(\boldsymbol{P})$ is a standard function, and consequently $\boldsymbol{B}(\boldsymbol{P})$ is standard function since minimum of two standard functions is a standard function [13]. Since the SNPC game admits at least one Nash equilibrium, $\boldsymbol{B}(\boldsymbol{P})$ has at least a fixed point, and the fixed point is unique since $\boldsymbol{B}(\boldsymbol{P})$ is a standard function [13].

\section{APPENDIX B}

In this Appendix, we show that the sequence of power updates generated by Algorithm 1 converge to the unique Nash equilibrium of the SNPC game $\left\langle\mathcal{N},\left\{\mathcal{P}_{i}\right\},\left\{U_{i}(\cdot)\right\}\right\rangle$. To this end, we show that the sequence $\left\{\boldsymbol{P}^{(n)}\right\}_{n=1}^{\infty}$, generated according to the fixed point iteration $\boldsymbol{P}^{(n+1)}=\boldsymbol{B}\left(\boldsymbol{P}^{(n)}\right)$ with $\boldsymbol{P}^{(1)}=\mathbf{0}$, is a convergent sequence.

Lemma 2: $\left\{\boldsymbol{P}^{(n)}\right\}_{n=1}^{\infty}$ is an increasing sequence.

Proof: We show this result by induction. Note that $\boldsymbol{P}^{(2)} \succ \boldsymbol{P}^{(1)}$. Assume that $\boldsymbol{P}^{(n)} \succeq \boldsymbol{P}^{(n-1)}$. Since $\boldsymbol{B}(\boldsymbol{P})$ is a standard function, we have $\boldsymbol{B}\left(\boldsymbol{P}^{(n)}\right) \succeq \boldsymbol{B}\left(\boldsymbol{P}^{(n-1)}\right)$ from monotonicity property which implies $\boldsymbol{P}^{(n+1)} \succeq \boldsymbol{P}^{(n)}$. Since $\left\{P_{i}^{(n)}\right\}_{\infty=1}^{\infty}$ is upper bounded, Lemma 2 implies that $\left\{\boldsymbol{P}^{(n)}\right\}_{n=1}^{\infty^{n=1}}$ is a convergent sequence [11]. Hence, $\left\{\boldsymbol{P}^{(n)}\right\}_{n=1}^{\infty}$ converges to the unique fix point of $\boldsymbol{B}(\boldsymbol{P})$ which is Nash equilibrium.

\section{APPENDIX C}

First, we rewrite (11) as the following fixed point equation

$$
\begin{aligned}
P_{i} & =\sqrt{\frac{\gamma_{\mathrm{th}}}{\mu_{i} G_{i i}^{s p}}}\left(\frac{\sigma^{2}}{G_{i i}^{s s}}+\sum_{j=1, j \neq i}^{N} \frac{G_{j i}^{s s}}{\frac{G_{i i}^{s s}}{P_{j}^{(n)}}+\frac{\gamma_{\mathrm{th}} G_{j i}^{s s}}{P_{i}}}\right)^{0.5} . \\
& =T_{i}\left(P_{i}, \boldsymbol{P}_{-i}^{(n)}\right)
\end{aligned}
$$

It is easy to show that $T_{i}\left(P_{i}, \boldsymbol{P}_{-i}^{(n)}\right)$ is a standard function with respect to $P_{i}$. Hence, if it has a fixed point it will be unique.

Lemma 3: $\left\{\pi_{m}(i)\right\}_{m=1}^{\infty}$ is an increasing sequence.

Proof: The proof is similar to that of Lemma 2, and is skipped to avoid repetition.

It is easy to show that $\left\{\pi_{m}(i)\right\}_{m=1}^{\infty}$ is bounded. Hence, Lemma 3 implies it is convergent. Since $T_{i}\left(P_{i}, \boldsymbol{P}_{-i}^{(n)}\right)$ is standard, $\left\{\pi_{m}(i)\right\}_{m=1}^{\infty}$ converges to the unique fixed point of (11).

\section{REFERENCES}

[1] A. Goldsmith, S. A. Jafar, I. Maric and S. Srinivasa, "Breaking spectrum gridlock with cognitive radios: an information theoretic perspective," Proc. IEEE, vol. 97, no. 5, pp. 894-914, May 2009.

[2] C. U. Saraydar, N. B. Mandayam and J. D. Goodman, "Efficient power control via pricing in wireless data networks," IEEE Trans. Commun., vol. 50, no. 2, pp. 291-303, Feb. 2002.

[3] T. Alpcan, T. Basar and S. Dey, "A power control game based on outage probabilities for multicell wireless data networks," IEEE Trans. Wireless Commun., vol. 5, no. 4, pp. 890-899, Apr., 2006.

[4] P. Dubey, "Inefficiency of Nash Equilibria", Mathematics of Operations Research, vol. 11, vo. 1, pp. 1-8, Feb., 1986.

[5] T. Alpcan and L. Pavel, "Nash equilibrium design and optimization," in Proc. Int. Conf. on Game Theory for Networks, pp. 164-170, May, 2009.

[6] Z. Pan, C. Yusun and J. A. Copeland, "Asynchronous Power Control Game with Channel Outage Constraints in Cognitive Radio Networks," in Proc. IEEE Int. Conf. on Communications, Jun. 2011.

[7] W. Yongle, B. Wang, K. J. R. Liu and T. C. Clancy, "Repeated open spectrum sharing game with cheat-proof strategies," IEEE Trans. Wireless Commun., vol. 8, no. 4, pp. 1922-1933, Apr., 2009.

[8] S. Kandukuri and S. Boyd, "Optima Power Control in InterferenceLimited Fading Wireless Channels with Outage-Probability Specifications," IEEE Trans. Wireless Commun., vol. 1, no. 1, pp. 46-55, Jan. 2002.

[9] S. Boyd and L. Vandenberghe, Convex Optimization, New York: Cambridge, 2004.

[10] N. D. Stein, Characterization and Computation of Equilibria in Infinite Games, Master Thesis, M.I.T., Jun., 2007.

[11] W. Rudin, Principles of Mathematical Analysis, New York: McGrawHill, Second edition, 1964.

[12] M. J. Osborne and A. Rubinstein, A course in game theory, Cambridge: MIT Press, 1994

[13] R. D. Yates, "A framework for uplink power control in cellular radio systems ", IEEE J. Sel. Areas Commun., vol. 13, no. 3, pp. 1341-1347, Sep. 1995. 